AperTO - Archivio Istituzionale Open Access dell'Università di Torino

\title{
Determination of radiation hardness of silicon diodes
}

\section{This is the author's manuscript}

Original Citation:

Availability:

This version is available http://hdl.handle.net/2318/1700238

since 2019-04-29T08:40:12Z

Published version:

DOI:10.1016/j.nimb.2019.04.032

Terms of use:

Open Access

Anyone can freely access the full text of works made available as "Open Access". Works made available under a Creative Commons license can be used according to the terms and conditions of said license. Use of all other works requires consent of the right holder (author or publisher) if not exempted from copyright protection by the applicable law. 


\title{
Determination of radiation hardness of silicon diodes.
}

E. Vittone $^{1}$, J. Garcia Lopez ${ }^{2}$, M. Jaksic ${ }^{3}$, M. C. Jimenez Ramos ${ }^{4}$, A. Lohstroh ${ }^{5}$, Z. Pastuovic ${ }^{6}$, S. Rath $^{7}$, R. Siegele ${ }^{6}$, N. Skukan ${ }^{3}$, G. Vizkelethy ${ }^{8 *}$, A. Simon ${ }^{9}$

${ }^{1}$ Physics Department and INFN, Torino Unit, via P. Giuria 1, 10125 Torino (I), ettore.vittone@unito.it

${ }^{2}$ Departamento de Física Atómica, Molecular y Nuclear. Facultad de Física. University of Sevilla, Spain

${ }^{3}$ Ruđer Bošković Institute, Zagreb, Croatia

${ }^{4}$ Centro Nacional de Aceleradores, University of Sevilla, Spain

${ }^{5}$ Department of Physics, University of Surrey, Guildford, Surrey, United Kingdom

${ }^{6}$ Center for Accelerator Science, ANSTO, 1 New Illawarra Rd, Lucas Heights NSW 2234, Australia

${ }^{7}$ Department of Physics \& Astrophysics., University of Delhi, Delhi-110007, India

${ }^{8 *}$ Sandia National Laboratories, Radiation-Solid Interactions, PO Box 5800, MS 1056, Albuquerque, NM 87185, USA

${ }^{6}$ International Atomic Energy Agency. Vienna International Center, PO Box 100, 1400 Vienna, Austria

Keywords: IBIC, radiation damage, radiation hardness, ion microbeam, silicon diode.

\begin{abstract}
In this paper, we describe an experiment aimed to measure the physical observables, which can be used for the assessment of the radiation hardness of commercially available silicon photo diodes commonly used as nuclear detectors in particle accelerator laboratories. The experiment adopted the methodology developed during the International Atomic Energy Agency (IAEA) Coordinated Research Project (CRP No. F11016) “Utilization of Ion Accelerators for Studying and Modelling Ion Induced Radiation Defects in Semiconductors and Insulators”.

This methodology is based on the selective irradiation of micrometer-sized regions with different

* This paper describes objective technical results and analysis. Any subjective views or opinions that might be expressed in the paper do not necessarily represent the views of the U.S. Department of Energy or the United States Government.Sandia National Laboratories is a multimission laboratory managed and operated by National Technology \& Engineering Solutions of Sandia, LLC, a wholly owned subsidiary of Honeywell International Inc., for the U.S. Department of Energy's National Nuclear Security Administration under contract DE-NA0003525.
\end{abstract}


fluences of $\mathrm{MeV}$ ions using an ion microbeam and on the measurement of the charge collection efficiency (CCE) degradation by Ion Beam Induced Charge (IBIC) microscopy performed in full depletion condition, using different probing ions.

The IBIC results are analyzed through a theoretical approach based on the Shockley-Read-Hall model for the free carrier recombination in the presence of ion-induced deep traps. This interpretative model allows the evaluation of the material radiation hardness in terms of recombination parameters for both electrons and holes.

The device under study in this experiment was a commercial p-i-n photodiode, which was initially characterized by i) standard electronic characterization techniques to determine its doping and ii) the Angle-Resolved IBIC to evaluate its effective entrance window. Nine regions of (100x100) $\mu \mathrm{m}^{2}$ were irradiated with $11.25 \mathrm{MeV} \mathrm{He}$ ions up to a maximum fluence of $3 \cdot 10^{12}$ ions $/ \mathrm{cm}^{2}$. The CCE degradation was measured by the IBIC technique using $11.25 \mathrm{MeV} \mathrm{He}$ and $1.4 \mathrm{MeV} \mathrm{He}$ as probing ions.

The model presented here proved to be effective for fitting the experimental data. The fitting parameters correspond to the recombination coefficients, which are the key parameters for the characterization of the effects of radiation damage in semiconductors.

\section{Introduction}

Semiconductor detectors are used in mixed radiation fields where more or less high degree of radiation tolerance is required: space and avionic applications [1], high energy physics experiments [2], medical diagnostic imaging and therapy, industrial imaging and material processing. In particle energy spectroscopy, the radiation tolerance of detectors is a major issue, due to the rapid degradation of detector performances as function of the ion fluence [3]. This issue is amplified further in activities involving the ion beam focused down to the micrometer-size spot, where high ion fluences can be easily obtained if selected regions of detectors are irradiated at the micrometer scale, even using relatively low ion currents. In this case, although localized irradiation induce negligible effects on global parameters, such as leakage current, it can strongly influence the charge collection efficiency of the detector.

A methodology aimed to measure the effective parameters affecting the degradation of electronic 
properties of a device is therefore of paramount importance for the assessment of the device lifetime and for the development of new devices with improved radiation hardness [4]. The new methodology including both the experimental protocol and the modeling has been recently developed within an IAEA Coordinated Research Project (CRP, reference F11016) "Utilization of Ion Accelerators for Studying and Modelling Ion Induced Radiation Defects in Semiconductors and Insulators" [5, 6]. The experimental protocol is based on the use of energetic accelerated ions in the $\mathrm{MeV}$ energy range, which play a dual role: (i) as damaging agents to generate defects in the active semiconductor material of the electronic device and (ii) as probes to measure the effect of the radiation damage on the Charge Collection Efficiency (CCE), as the main feature defining the electronic response of the tested/irradiated semiconductor devices. The model is valid for low level damage, which is assumed to be an accumulation of simple point defects (vacancies, di-vacancies, interstitials or their complexes with atoms present in the crystal lattice) created in ion cascades. We assume that the subsequent cascades are separated either in space or time so there is no interaction between them. Focused ion microbeams and the Ion Beam Induced Charge $[7,8]$ technique are required in both phases of the experiment including (i) the creation of patterned partly damaged areas covering the large range of implantation fluences and (ii) measuring of the deterioration of CCE due to created damage. One advantage of this methodology for evaluation of the radiation hardness in semiconductor materials is that the increase of dark current affecting device features is negligible, due to the small size of the damaged region compared to the whole active volume of the device. This is an important factor, which allows IBIC to be employed as a very effective characterization technique for the functional analysis of electronic materials, both for the solid theoretical foundations, which provide effective tools for data analysis, and the easiness of implementation to any nuclear microprobe facility. In this paper, the above mentioned experimental methodology [6] has been adopted to extract key parameters for the characterization of the effects of radiation damage in a commercially available silicon photodiode commonly used in ion beam laboratories. These parameters are the recombination coefficients, which include the average number of stable radiation induced defects generated by irradiation. The experiment was performed in the low level of damage regime, which induces a linear degradation of the charge collection efficiency. Such a condition allows the final fitting algorithms to be linearized, which leads to a significant simplification of the algorithms for analysis of the 
experimental data.

\section{Experimental}

The sample under study was a commercial silicon photo-diode, Hamamatsu S1223 commonly used in IBA laboratories $[9,10]$. The metal can package was opened by removing the borosilicate glass window in order to expose the frontal surface of the photodiode to incident $\mathrm{MeV}$ ions [11]. Since the photodiode is photon-sensitive, all the measurements were carried out in dark conditions to avoid the contribution of the photocurrent to the electronic noise.

Almost constant concentration of donors in the base of about $7 \cdot 10^{13} \mathrm{~cm}^{-3}$ was calculated from capacitance-voltage measurements; the $\mathrm{p}^{+}$layer thickness of about $0.6 \mu \mathrm{m}$ with a maximum concentration of acceptor of $2 \cdot 10^{19} \mathrm{~cm}^{-3}$ was estimated by spreading resistance profiling. These data were used for modeling the electrostatics and the transport properties of the diode by solving the Poisson's and the continuity equations [12] by means of Finite Element Methods [13]. Figure 1 shows the main quantities resulting from this analysis: the Gunn's weighting field and the carrier drift velocity profiles at a reverse bias voltage of $100 \mathrm{~V}$.

The preliminary analysis of the sample under study was completed by measurement of the effective entrance window (or dead layer) thickness by the angle resolved IBIC analysis (ARIBIC) $[14,15,16]$. The inset of Figure 2 shows a schematics of the measurement, which was performed at the ion microbeam facility of Ruder Boskovic Institute [17], using Li ions with energy Eion=2.28 MeV. The measurement was carried out in full depletion condition: the applied bias voltage of $20 \mathrm{~V}$ induces a depletion layer width of $60 \mu \mathrm{m}$, which is much larger than the Li ion range in $\mathrm{Si}(5.2 \mu \mathrm{m})$.

As shown in Fig. 2, the diode's response at different angle of incidence $\theta$ decreases due to the increase of the energy loss of ion in the dead layer of thickness $t^{*}$. Assuming that all carriers generated in the depletion region induce a charge, which is totally collected, the measured pulse height $\mathrm{Q}(\theta)$ at the incidence $\theta$ normalized to the pulse height measured for normal incidence $(\theta=0)$, is given by:

$$
\text { (1) } \frac{\mathrm{Q}(\theta)}{\mathrm{Q}(\theta=0)}=1-\left.\frac{1}{E_{\text {ion }}} \frac{\mathrm{d} E}{\mathrm{~d} x}\right|_{0} t^{*} \cdot\left(\frac{1}{\cos (\theta)}-1\right)
$$

The value of the term $\left.\frac{1}{E_{\text {ion }}} \frac{\mathrm{d} E}{\mathrm{~d} x}\right|_{0} t^{*}=(0.0407 \pm 0.0012)$ is extracted from the fit of experimental data using eq. (1). From SRIM2013 simulation [18], the electronic stopping power of $2.28 \mathrm{MeV} \mathrm{Li}$ 
ions in silicon is $\left.\frac{d E}{d x}\right|_{0}=513 \mathrm{eV} / \mathrm{nm}$; therefore $\mathrm{t}^{*}=180 \mathrm{~nm}$. This value is to be considered the effective thickness of the dead layer in silicon, which includes both the top oxide layer (around 110 $\mathrm{nm}$, as measured by Rutherford Backscattering Spectroscopy (RBS) ) and the thickness in silicon immediately beneath the electrode ( $p+$ layer) in which charge collection is inefficient.

We adopted the protocol described in [6] to assess the radiation hardness of the diode. Nine distinct (100x100) $\mu \mathrm{m}^{2}$ regions of the diode under study were irradiated at increasing fluences of $11.25 \mathrm{MeV}$ He ions (in the following named damaging ion beam = DIB) at the ANSTO ion microprobe facility [19].

The effect of the radiation damage was probed using 1.4 MeV and 11.25 MeV He ion microbeam (in the following named Probing Ion Beams = PIBs); the IBIC measurements were carried out at a constant applied bias voltage of $100 \mathrm{~V}$, in order to fulfil the full depletion condition. Since the characterization phase was carried out a few days (during which the sample was kept at room temperature) after irradiation, only permanent damage induced by the displacement of atomic nuclei is considered. The median CCE relevant to the central part of the irradiated regions are shown in Figure 3 as function of the DIB fluence.

\section{Data analysis}

The experiment was designed to meet the assumptions of the model described in [6]. The IBIC measurements were performed at $100 \mathrm{~V}$ and the depletion layer (depth of about $150 \mu \mathrm{m}$ as shown in Fig. 1) extends beyond the range of $1.4 \mathrm{MeV}(\approx 5 \mu \mathrm{m})$ and $11.25 \mathrm{MeV}(\approx 85 \mu \mathrm{m})$ He ions, as shown in Fig. 4.

Under these full depletion conditions, we can assume that the dominant charge transport mechanism is the carrier drift caused by the applied electric field whose direction is perpendicular to the electrodes and that all carrier recombination processes caused by ion damage occur within the depletion region of the device.

Assuming complete collection of the induced charge (i.e. CCE $=100 \%$ ) in the pristine diode and adopting the Shockley-Read-Hall approach to model the carrier lifetime as function of the recombination center concentration, which is assumed to be proportional to the concentration of vacancy-interstitial Frenkel pairs created by DIB irradiation, the CCE degradation can be expressed 
through the following expression:

$$
\text { (2) } \mathrm{CCE}=\int_{0}^{d} \mathrm{~d} x \quad \gamma(x)\left\{\begin{array}{c}
\int_{x}^{d}\left\{E^{+}(y) \exp \left[-\alpha_{\mathrm{e}} \cdot \Phi \int_{x}^{y} \mathrm{~d} z \frac{\eta(z)}{v_{\mathrm{e}}(z)}\right]\right\} \mathrm{d} y \\
+ \\
\int_{0}^{x}\left\{E^{+}(y) \exp \left[-\alpha_{\mathrm{h}} \cdot \Phi \int_{y}^{x} \mathrm{~d} z \frac{\eta(z)}{v_{\mathrm{h}}(z)}\right]\right\} \mathrm{d} y
\end{array}\right\}
$$

where $\gamma=\frac{1}{E_{\mathrm{I}}} \frac{\mathrm{d} E_{\mathrm{I}}}{\mathrm{d} x}$ is the normalized ionization energy loss profile of the PIBs, $\eta$ is the vacancy profile (the number of vacancies generated per ion per unit length, see Fig. 4) as simulated by SRIM, $\Phi$ is the DIB fluence, $v_{\mathrm{e}, \mathrm{h}}$ are the drift velocities of electrons (subscript e) and holes (subscript h). The term $\mathrm{E}^{+}=\frac{\partial \mathrm{E}}{\partial \mathrm{V}}$ is Gunn's weighting field defined as the partial derivative of the actual electric field (E) with respect to the bias voltage $\mathrm{V}$ applied to the sensing electrode, while the other electrode is grounded. Gunn's weighting potential $\varphi^{+}=\frac{\partial \varphi}{\partial V}$ is similarly defined, where $\varphi$ is the actual electrostatic potential.

Finally, the terms

(3) $\alpha_{\mathrm{e}, \mathrm{h}}=k_{\mathrm{e}, \mathrm{h}} \cdot \sigma_{\mathrm{e}, \mathrm{h}} \cdot v_{e, h}^{t h}$

are the capture (or recombination) coefficients, as usually defined in the Shockley-Read-Hall model: $v_{e, h}^{t h}$ and $\sigma_{\mathrm{e}, \mathrm{h}}$ are the carrier thermal velocities and the carrier capture cross sections, respectively. The coefficients $k_{\mathrm{e}, \mathrm{h}}$ represent the average number of active defects (carrier traps) generated by a single (SRIM simulated) vacancy.

The low level of damage investigated in this experiment, induces a linear degradation of the CCE, as is shown in Fig. 3. Therefore, the exponentials in Eq. (2) can be expanded in Taylor series, providing the following approximate expression:

(4) $\mathrm{CCE}=1-\alpha_{\mathrm{e}} \cdot \Phi_{e}^{*}-\alpha_{\mathrm{h}} \cdot \Phi_{h}^{*}$

Where we have defined the effective fluences for electrons and holes as follows

(5)

$$
\begin{gathered}
\Phi_{e}^{*}=\Phi \cdot \int_{0}^{d} \mathrm{~d} x \frac{\eta(x)}{v_{\mathrm{e}}(x)} \cdot \varphi^{+}(x) \int_{0}^{x} \mathrm{~d} z \quad \gamma(z) \\
\Phi_{h}^{*}=\Phi \cdot \int_{0}^{d} \mathrm{~d} x \frac{\eta(x)}{v_{\mathrm{h}}(z)} \cdot\left[1-\varphi^{+}(x)\right] \int_{x}^{d} \mathrm{~d} z \quad \gamma(z)
\end{gathered}
$$

Being, by definition

$$
\text { (6) } \int_{0}^{d} \gamma(x) \mathrm{d} x=\int_{0}^{d} E^{+}(y) \mathrm{d} y=1
$$

Since all terms in the integrals of eq. (5) can be calculated, ( $\eta$ and $\gamma$ are known from SRIM simulations (see Fig. 4) and $v_{\mathrm{e}, \mathrm{h}}, \varphi^{+}$are calculated from the device modeling (see Fig. 1) ), a 
multivariate linear regression can be used in order to evaluate the parameters $\alpha_{\mathrm{e}}$ and $\alpha_{\mathrm{h}}$, which determine the "best" straight lines through the measured points [20]. For this purpose, the vector of the unknown

(7) $\vec{\alpha}=\left(\begin{array}{c}\alpha_{\mathrm{e}} \\ \alpha_{\mathrm{h}}\end{array}\right)$ can be evaluated through the following matrix equation:

(8) $\vec{\alpha}=\left(A^{T} \cdot G \cdot A\right)^{-1} A^{T} \cdot G \cdot \mathbb{C}$

Where $\mathrm{G}$ is $(2 \times \mathrm{NN}) \mathrm{x}(2 \mathrm{xN})$ diagonal weight matrix $G_{i, i}=1 / \sigma_{i}^{2}$ and

$$
\text { (9) } A=\left(\begin{array}{cc}
{ }^{P I B 1} \Phi_{e, 1}^{*} & { }^{P I B 1} \Phi_{h, 1}^{*} \\
{ }^{P I B 1} \Phi_{e, 2}^{*} & { }^{P I B 1} \Phi_{h, 2}^{*} \\
\ddot{{ }^{P I B 1}} \Phi_{e, \mathrm{~N}}^{*} & { }^{P I B 1} \Phi_{h, \mathrm{~N}}^{*} \\
{ }^{P I B 2} \Phi_{e, 1}^{*} & { }^{P I B 2} \Phi_{h, 1}^{*} \\
{ }^{P I B 2} \Phi_{e, 2}^{*} & { }^{P I B 2} \Phi_{h, 2}^{*} \\
{ }^{P I B 2} \Phi_{e, \mathrm{~N}}^{*} & { }^{P I B 2} \Phi_{e, \mathrm{~N}}^{*}
\end{array}\right) ; \mathbb{C}=\left(\begin{array}{c}
1-{ }^{P I B 1} C C E_{1} \\
1-{ }^{P I B 1} C C E_{2} \\
1-\stackrel{{ }^{P I B 1} C C E_{\mathrm{N}}}{1-{ }^{P I B 2} C C E_{1}} \\
1-{ }^{P I B 2} C C E_{2} \\
1-{ }^{P I B 2} C C E_{\mathrm{N}}
\end{array}\right)
$$

$\mathrm{N}(=9)$ is the number of experimental data relevant to a single PIB as reported in Fig. 3.

The covariance $\mathrm{C}$ matrix of the best estimates $\vec{\alpha}$ is given by:

(10) $\quad C=\left(A^{T} \cdot G \cdot A\right)^{-1}$

The solution of eq. (8) leads to:

$$
\vec{\alpha}=\left(\begin{array}{l}
\alpha_{\mathrm{e}}=1500 \mu \mathrm{m}^{3} / \mathrm{s} \\
\alpha_{h}=7800 \mu \mathrm{m}^{3} / \mathrm{s}
\end{array}\right)
$$

And the covariance matrix is given by:

(12) $C=10^{6} \cdot\left(\begin{array}{cc}0.22 & -0.77 \\ -0.77 & 3.4\end{array}\right)$

The results of the fitting procedure are shown in Fig. 3 (solid lines). The uncertainties of the parameters $\alpha_{\mathrm{e}}$ and $\alpha_{\mathrm{h}}$, correspond to the covariance ellipse shown in Fig. 5.

\section{Discussion and conclusions}

As already mentioned, the experiment described here was designed in order to fulfil all the conditions which underpin the model proposed in [6]. In particular, we confined the analysis to low fluence ion irradiation, in order to avoid any significant alteration of the basic device properties (e.g. the effective doping density, which modify the electrostatic features of the pristine diode). This low damage regime allows the basic theory of charge induction to be applied using the electrostatics and the transport 
parameters (namely the carrier velocities and the Gunn's potential) evaluated through the solution of the standard semiconductor equations, which are based on the pristine doping profile extracted from Capacitance-Voltage and spreading resistance measurements. To complete the evaluation of the active region of diode, ARIBIC analysis was performed in order to measure the dead layer underlying the front electrode.

Moreover, the low level damage approximation allows the usage of the Shockley-Read-Hall recombination model for free carrier lifetimes, which depend on displacement damage, which is assumed to be proportional to the distribution of primary point defects acting as recombination centers.

Under full depletion conditions, the basic IBIC equations can be analytically solved. The resulting expression of the CCE incorporates terms relevant to the electrostatic features of the diode, the vacancy profile induced by DIB and the carrier generation profile produced by PIBs.

In this study, we used one shallow (1.4 MeV He) and one deep-penetrating (11.25 MeV He) PIB, in order to investigate the effect of damage on the recombination of both the carriers (electrons and holes).

In the low damage regime, the CCE dependence of the DIB fluence (eq. 6) can be linearized, providing a simpler approach to fit the experimental data. The slopes of the fitting lines resulting from a bivariate linear procedure are the two recombination coefficients $\left(\alpha_{\mathrm{e}}\right.$ and $\left.\alpha_{\mathrm{h}}\right)$, which are indicators of the radiation hardness of a semiconductor material. Actually they (eq. (3) ) depend on (i) the capture cross sections $\sigma_{\mathrm{e}, \mathrm{h}}$, which identify the nature of the recombination centers and (ii) the parameters $k e, \mathrm{~h}$, which are proportionality factors relating active recombination centers with the concentration of Frenkel pairs introduced as primary point defects, the latter being predicted by SRIM.

The results of this analysis are summarized in eq. (11) and in Fig. 6. Assuming carrier thermal velocities values of $v_{\mathrm{e}}^{\text {th }}=2.05 \cdot 10^{7} \mathrm{~cm} / \mathrm{s}$ and $v_{\mathrm{h}}^{\text {th }}=1.69 \cdot 10^{7} \mathrm{~cm} / \mathrm{s}[21]$ the $k_{e, h} \sigma_{e, h}$ products derived from Eq. (3) are $k_{\mathrm{e}} \sigma_{\mathrm{e}}=7.4 \cdot 10^{-17} \mathrm{~cm}^{2}$ and $k_{\mathrm{h}} \cdot \sigma_{\mathrm{h}}=4.6 \cdot 10^{-16} \mathrm{~cm}^{2}$ for electrons and holes, respectively. If a single acceptor state of divacancy is assumed to be the most abundant electronically active defect in the high purity and low doped n-type silicon created by ion irradiation [22], the capture cross sections are $\sigma_{\mathrm{e}}=5 \cdot 10^{-15} \mathrm{~cm}^{-2}$ and $\sigma_{\mathrm{h}}=5 \cdot 10^{-14} \mathrm{~cm}^{-2}$ [23], for electrons and holes, respectively. Therefore, the relevant $k$-term values are $k e \approx 1.5 \cdot 10^{-2}$ and $k \mathrm{~h} \approx 1.0 \cdot 10^{-2}$, i.e. about 67 and 100 radiation induced 
defects are required to form one stable electron and hole recombination center, respectively.

The covariance ellipse shown in Fig. 5, provides information on the parameter uncertainties. The principal axes of the ellipse have an angle of about $13^{\circ}$ with respect to the $\alpha_{\mathrm{h}}$ axis, which indicates a correlation between the two recombination coefficients. This can be attributed to the definitions of the effective fluences (eq. (5) ), which involve common terms given by the normalized charge generation profiles $\gamma$, the vacancy distribution $\eta$ and the weighting potential $\varphi^{+}$. Therefore, the final general expression Eq. (4) naturally correlates the two contributions. The major axis of the ellipse is mainly related to the uncertainty of the hole recombination coefficient $\alpha_{\mathrm{h}}$. Actually, the hole trapping contribution to the CCE degradation can be ascribed only to measurements carried out with $\mathrm{PIB}=11.25 \mathrm{MeV} \mathrm{He}$, whose generation profile cover the damage profile (see Fig. 4), rapidly decreasing at the vacancy peak. Therefore, holes (moving towards the cathode (front electrode) located at $\mathrm{x}=0$ ) partially suffer recombination at the highly damaged region and their contribution to the CCE degradation is relatively small and subjected to high uncertainty. On the other hand, electrons move left to right across the depletion region, and for both the PIBs they cross the highly damaged region, providing the dominant contribution to the CCE degradation.

Finally, it is worth noting the main limited validity of the results presented in this work. As previously stated [6], the vacancy profile $\eta$ used in this work is presumably underestimated, owing to the limitations of the binary collision approximation, which is at the basis of SRIM simulations, neglecting both the crystal structure and the diffusion of initially generated displacement defects. Therefore, these results can be unreliable for more detailed investigations on the nature of stable radiation induced defects.

However, assuming that SRIM provides realistic but un-normalized vacancy and ionization profiles, the capture coefficients extracted from the fitting procedure can be considered reference values, which can be used to compare the radiation hardness of different semiconductor materials or devices and to envisage the corresponding CCE degradation for any ion irradiation and bias conditions.

\section{Acknowledgements}

This work has been carried out within the IAEA coordinated research project F11016. "Utilization of Ion Accelerators for Studying and Modelling of Radiation Induced Defects in Semiconductors and 
Insulators". It has been in part supported by Croatian Science Foundation under the project MIOBICC (No. 8127). EV acknowledges support from the project "Departments of Excellence" (L. 232/2016), funded by the Italian Ministry of Education, University and Research (MIUR) and from the experiment "ASIDI" of the Italian Institute for Nuclear Physics (INFN). The support by Australian government through the NCRIS funding scheme is acknowledged as well.

\section{References}

[1] A. Rehman, S.H. Lee, S.H. Lee, "Silicon space solar cells: progression and radiation-resistance analysis", Journal of the Korean Physical Society, 68(4), pp. 593-598 (2016)

[2] RD50 COLLABORATION, Radiation hard semiconductor devices for very high luminosity colliders (2018), https://rd50.web.cern.ch/rd50/.

[3] G. Kalinka, M. Novak, A. Simon, Z. Pastuovic, M. Jaksic, A.Z. Kiss, "Empirical approach to the description of spectral performance degradation of silicon photodiodes used as particle detectors", Nuclear Instruments \& Methods in Physics Research Section B 267, 2203-2207 (2009).

[4] S.R. Messenger, E.A. Burke, G. P. Summers, R.J. Walters, J. H. Warner, “Criteria for identifying radiation resistant semiconductor materials", IEEE Trans. on Nucl. Science, 52, 2276-2280 (2005).

[5] E. Vittone, A. Simon, M.B.H. Breese, "Papers arising from IAEA Coordinated Research Project "Utilization of ion accelerators for studying and modelling of radiation induced defects in semiconductors and insulators" (F11016)", Nuclear Instruments and Methods in Physics Research B $372(2016) 127$

[6] E. Vittone, Z. Pastuovic, M.B.H. Breese, J. Garcia Lopez, M. Jaksic, J. Raisanen, R. Siegele, A. Simon, G. Vizkelethy, "Charge collection efficiency degradation induced by $\mathrm{MeV}$ ions in semiconductor devices: Model and experiment ", Nuclear Instruments \& Methods in Physics Research Section B 372 (2016) 128-142.

[7] M.B.H. Breese, E. Vittone, G. Vizkelethy, P.J. Sellin, A review of ion beam induced charge microscopy, Nuclear Instruments \& Methods in Physics Research Section B 264 345-360, (2007).

[8] E. Vittone, Z. Pastuovic, O. Olivero, C. Manfredotti, M. Jaksic, A. Lo Giudice, F. Fizzotti, E. Colombo, "Semiconductor characterization by scanning ion beam induced charge (IBIC) 
microscopy”, Nuclear Instruments \& Methods in Physics Research Section B 266 (2008) 1312 1318.

[9] A. Simon, G. Kalinka, "Investigation of charge collection in a silicon PIN photodiode". Nuclear Instruments \& Methods in Physics Research Section B 231 507, (2005).

[10] D. Spemann, T. Reinert, J. Vogt, T. Andrea, N. Barapatre, R. Feder, A.M. Jakob, N. Liebing, C. Meinecke, F. Menzel, M. Rothermel, T. Butz, "Materials analysis and modification at LIPSION Present state and future developments", Nuclear Instruments \& Methods in Physics Research Section B 269 2175-2179, (2011).

[11] M.B.H. Breese, D.N. Jamieson, J.P.C. King, "Materials analysis using a nuclear microprobe", John Wiley Sons (1996).

[12] S. Selberherr, “Analysis and simulation of semiconductor devices”, Springer Verlag Wien, (1984).

[13] COMSOL, Multiphysics Modelling and Simulation, ver. 3.5. (2018) www.comsol.com.

[14] G.F. Knoll, "Radiation Detection and Measurement", John Wiley \& Sons Inc; 4th Ed. (2010), ISBN: 978-0470131480.

[15]A. Lo Giudice, F. Fizzotti, C. Manfredotti, E. Vittone, F. Nava, "Average energy dissipated by mega-electron-volt hydrogen and helium ions per electron-hole pair generation in 4H-SiC", Applied Physics Letters 87, 222105 (2005).

[16]A. Lo Giudice, Y. Garino, C. Manfredotti, V. Rigato, E. Vittone "Angle resolved IBIC analysis of 4H-SiC Schottky diodes", Nuclear Instruments and Methods in Physics Research B 249 213-216 (2006).

[17] M. Jakšić, I. Bogdanovic-Radovic, M. Bogovac, V. Desnica, S. Fazinic, M. Karlusic, Z. Medunic, H. Muto, Z. Pastuovic, Z. Siketic, N. Skukan, and T. Tadic, "New capabilities of the Zagreb ion microbeam system", Nuclear Instruments and Methods in Physics Research B 260, 114118 (2007).

[18] J.F: Ziegler, M.D. Ziegler, J.P. Biersack, "SRIM - The stopping and range of ions in matter." Nuclear Instruments and Methods in Physics Research B 268 11-12 1818(2010). 
[19] R. Siegele, D.D. Cohen, N. Dytlewski, "The ANSTO high energy heavy ion microprobe", Nuclear Instruments and Methods in Physics Research, Section B 158(1-4), pp. 31-38 (1999).

[20] S. Brandt, "Statistical and computational methods in data analysis", North Holland Publishing Company, Amsterdam, 1970.

[21] J.S. Laird, R.A.Bardos, C.Jagadish, D.N. Jamieson, G.J.F. Legge, "Scanning ion deep level transient spectroscopy”. Nuclear Instruments and Methods in Physics Research, Section B 158 1-4 464(1999).

[22] Z. Pastuovic' , E. Vittone, I. Capan, M. Jakšic' , Probability of divacancy trap production in silicon diodes exposed to focused ion beam irradiation, Appl. Phys. Lett. 9824 (2011).

[23] L. Vines, E. V. Monakhov, J. Jensen, A. Yu. Kuznetsov, B. G., "Effect of spatial defect distribution on the electrical behavior of prominent vacancy point defects in swift-ion implanted Si." Phys. Rev. B 7971 (2009). 
Figure 1: Left scale: Electron $\left(\mathrm{v}_{\mathrm{e}}\right) /$ hole $\left(\mathrm{v}_{\mathrm{h}}\right)$ velocity .Right scale: Gunn's weighting potential $\left(\varphi^{+}\right)$ profiles at a bias voltage of $100 \mathrm{~V}$.

Figure 2: Peak channel vs. tilting angle: experimental data (markers) and fit (solid curve) by means of Eq. (1).Inset: Scheme of the Angle-Resolved IBIC experiment to evaluate the entrance window thickness.

Figure 3: CCE degradation as function of the $\mathrm{DIB}=11.25 \mathrm{MeV}$ He fluence and probed with $1.4 \mathrm{MeV}$ $\mathrm{He}$ and 11.25 MeV PIBs. Markers represent experimental data and the solid lines result from the fitting procedure described in the text.

Figure 4: Left axis: ionization energy loss for different PIBs in silicon calculated by SRIM simulations. Right axis: vacancy profile (blue curve) of 11.25 MeV He ions in Si. All the profiles were calculated by SRIM simulations.

Figure 5: covariance ellipse of the fitting quantities $\alpha_{e}$ and $\alpha_{h}$. 


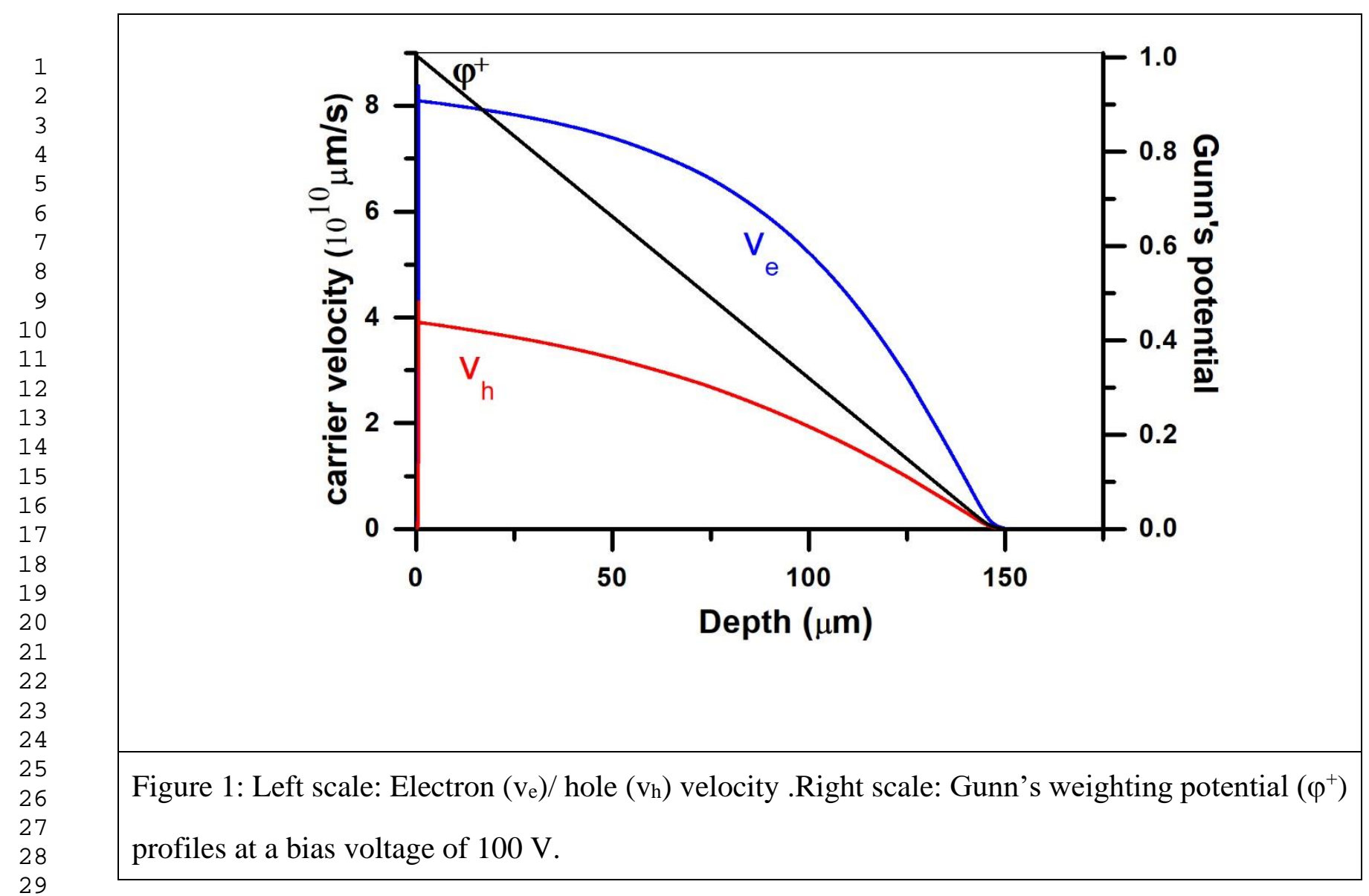




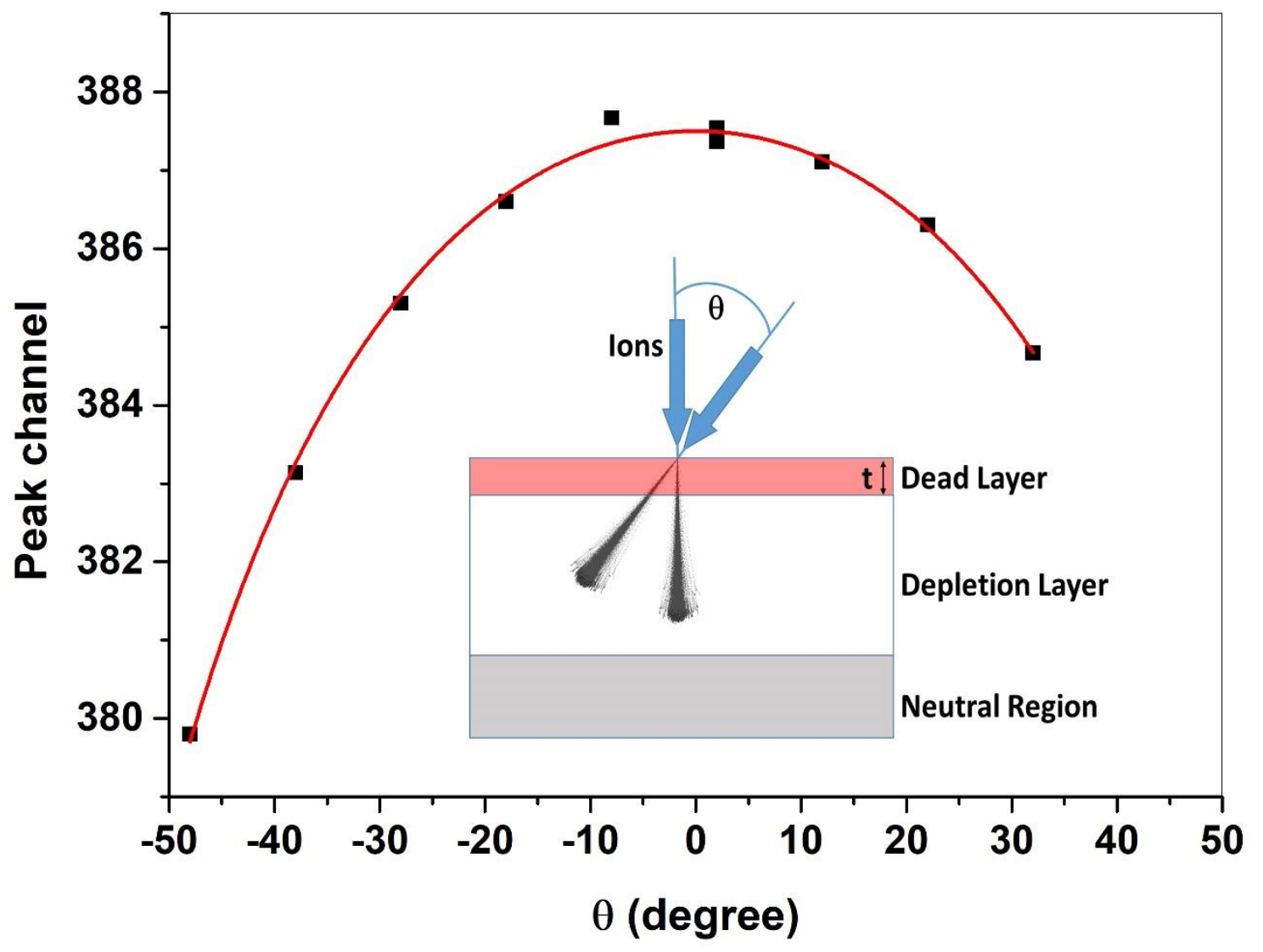

Figure 2: Peak channel vs. tilting angle: experimental data (markers) and fit (solid curve) by means of Eq. (1). Inset: Scheme of the Angle-Resolved IBIC experiment to evaluate the entrance window thickness. 


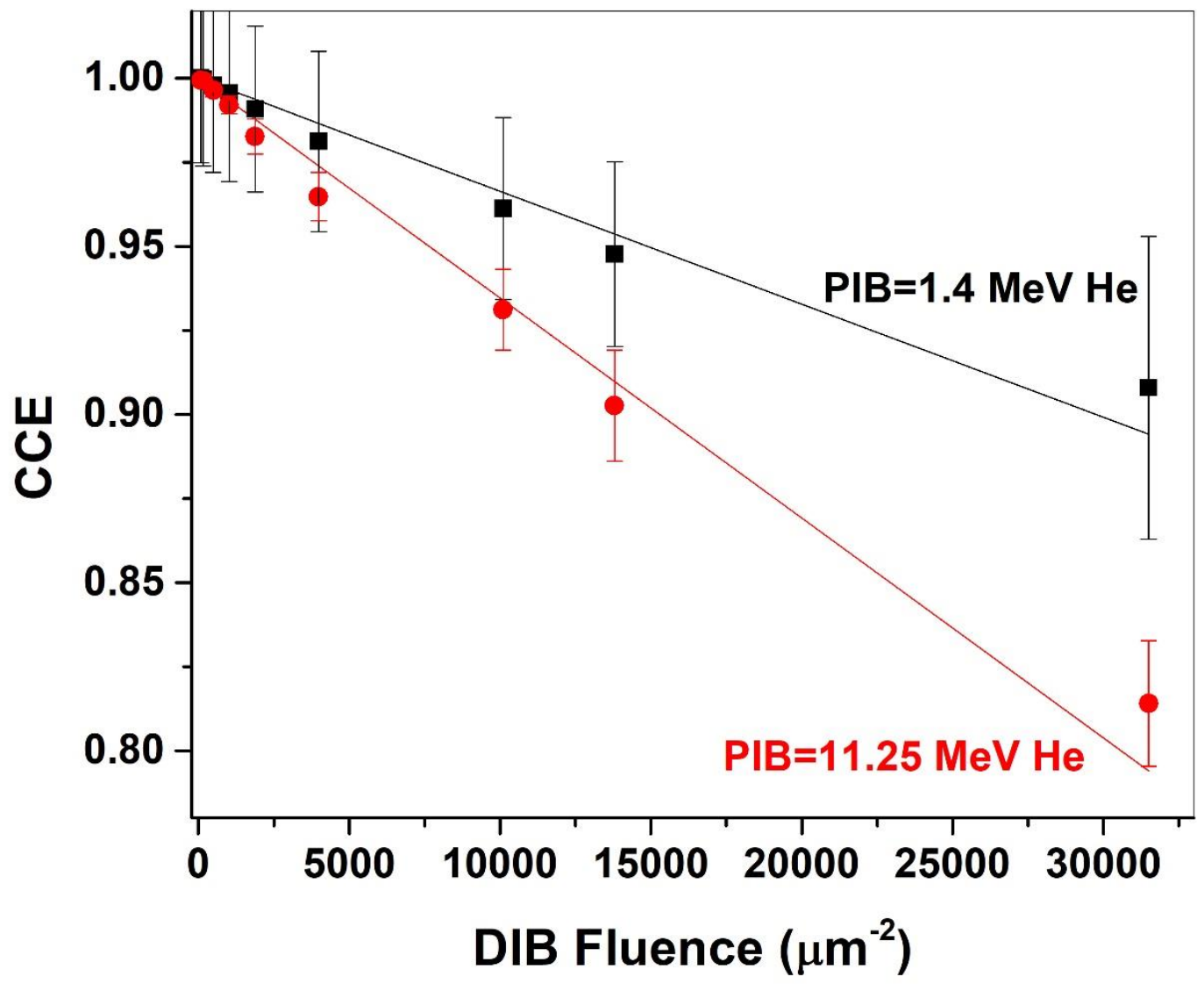

Figure 3: CCE degradation as function of the $\mathrm{DIB}=11.25 \mathrm{MeV}$ He fluence and probed with 1.4 $\mathrm{MeV} \mathrm{He}$ and 11.25 MeV PIBs. Markers represent experimental data and the solid lines result from the fitting procedure described in the text. 


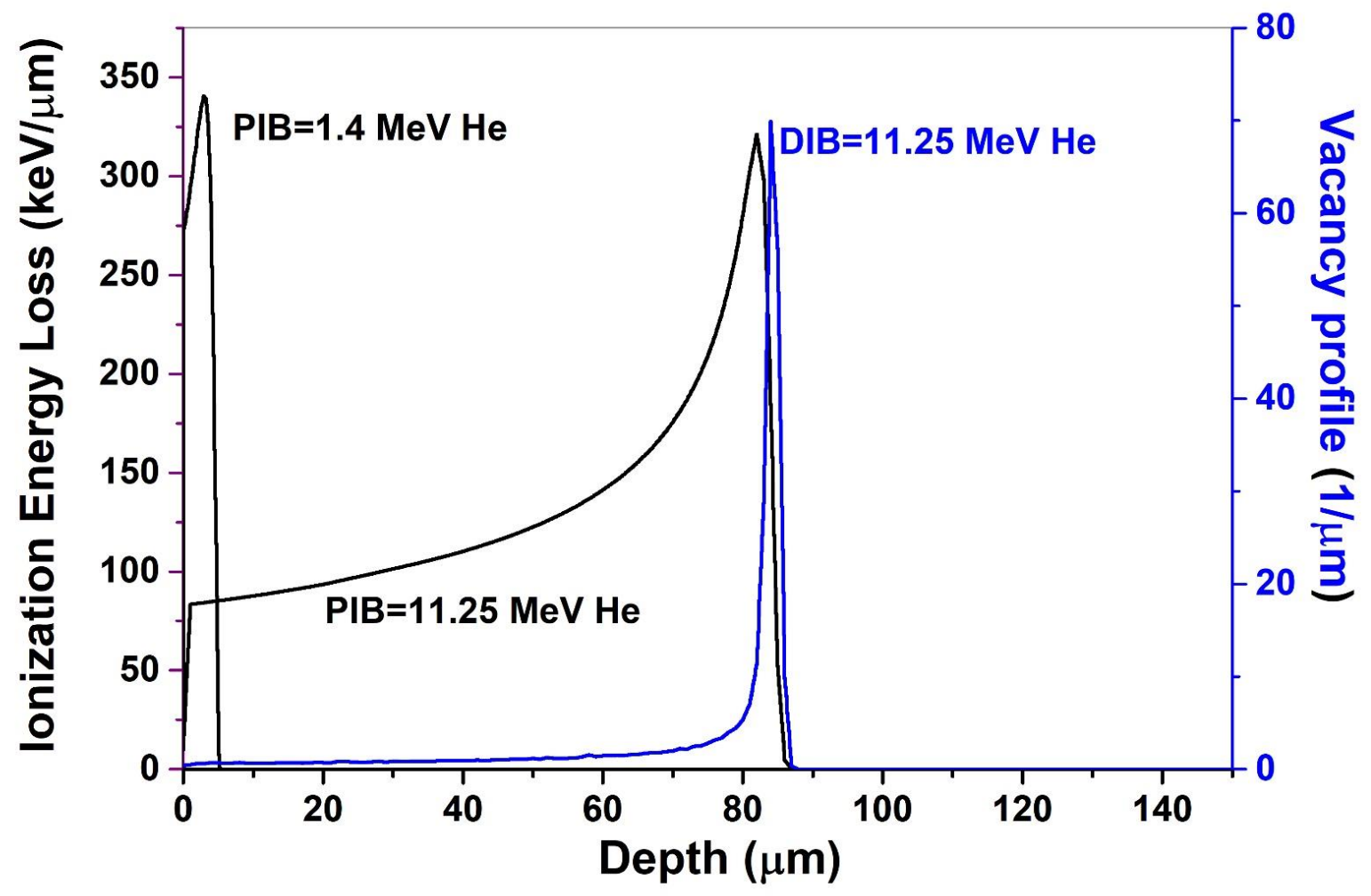

Figure 4: Left axis: ionization energy loss for different PIBs in silicon calculated by SRIM simulations. Right axis: vacancy profile (blue curve) of $11.25 \mathrm{MeV} \mathrm{He}$ ions in $\mathrm{Si}$. All the profiles were calculated by SRIM simulations. 


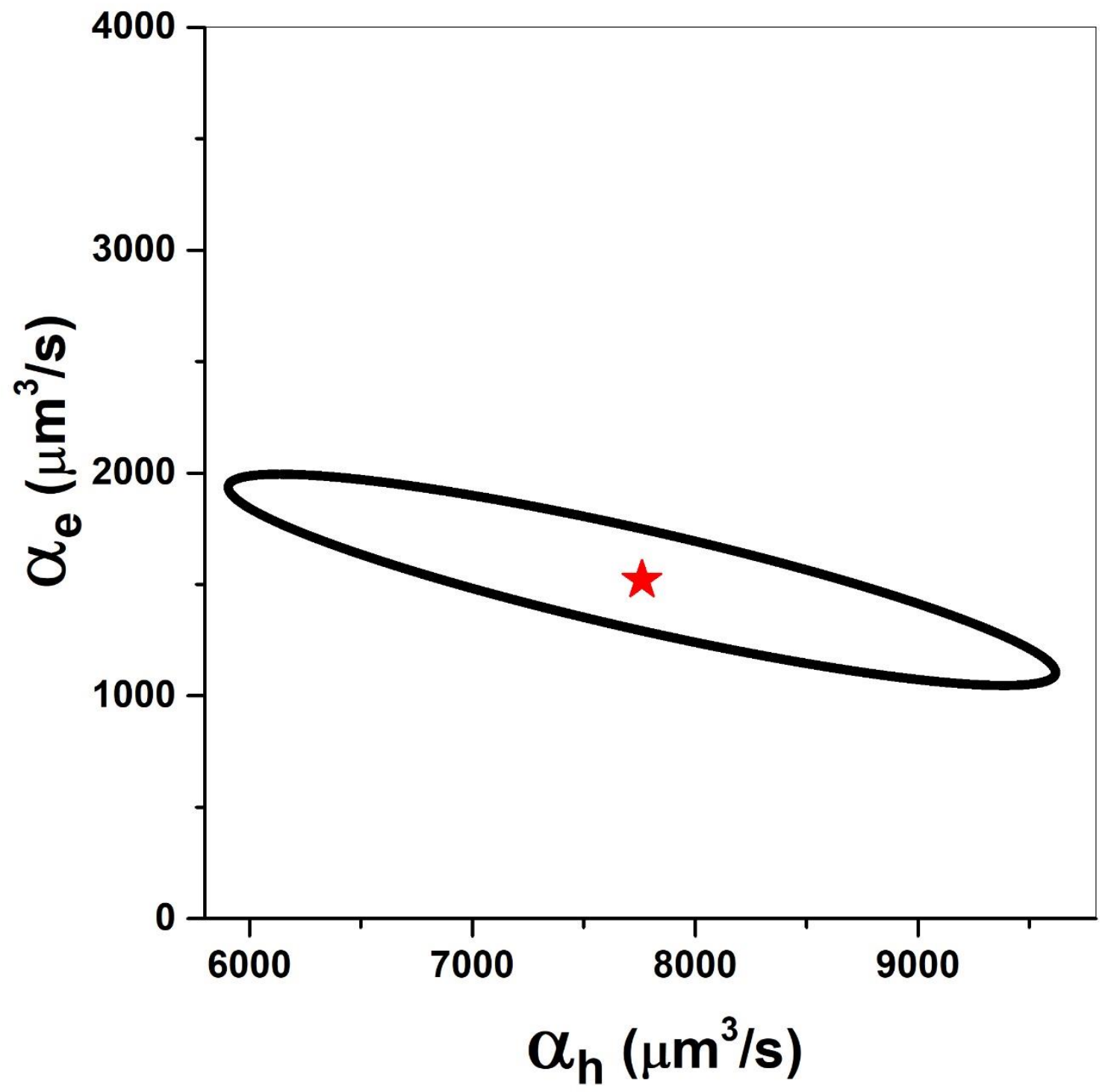

Figure 5: covariance ellipse of the fitting quantities $\alpha_{\mathrm{e}}$ and $\alpha_{\mathrm{h}}$. 


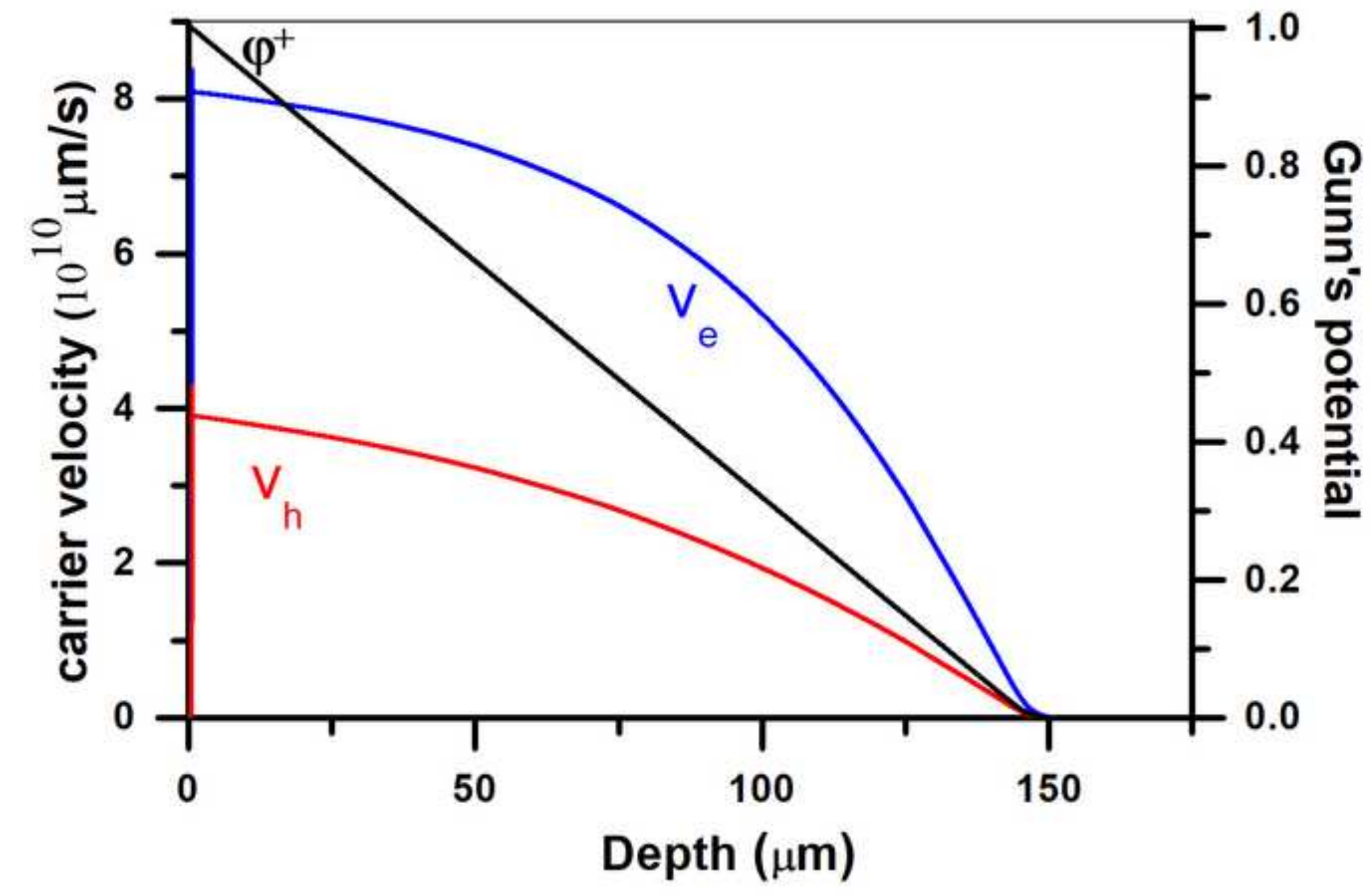




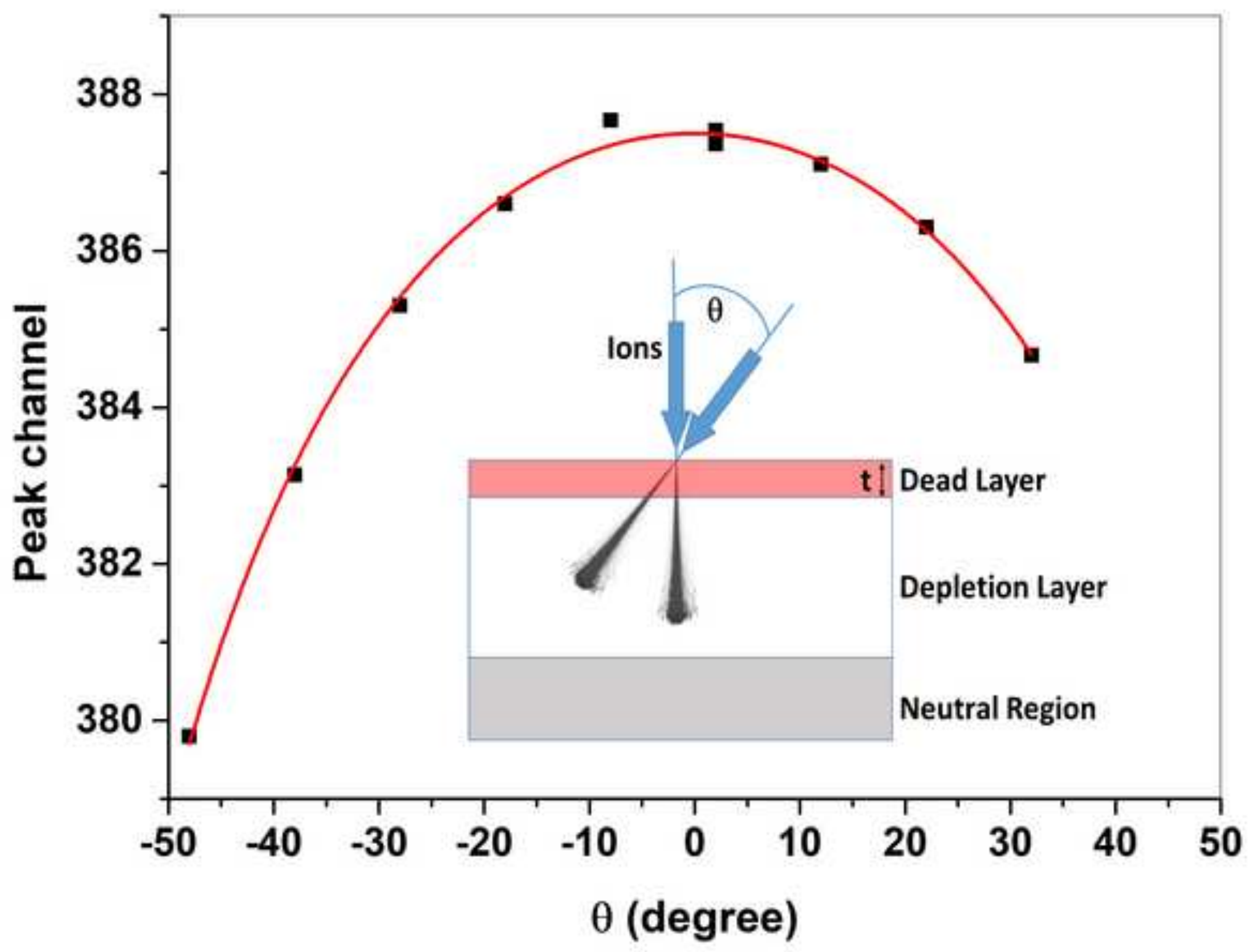




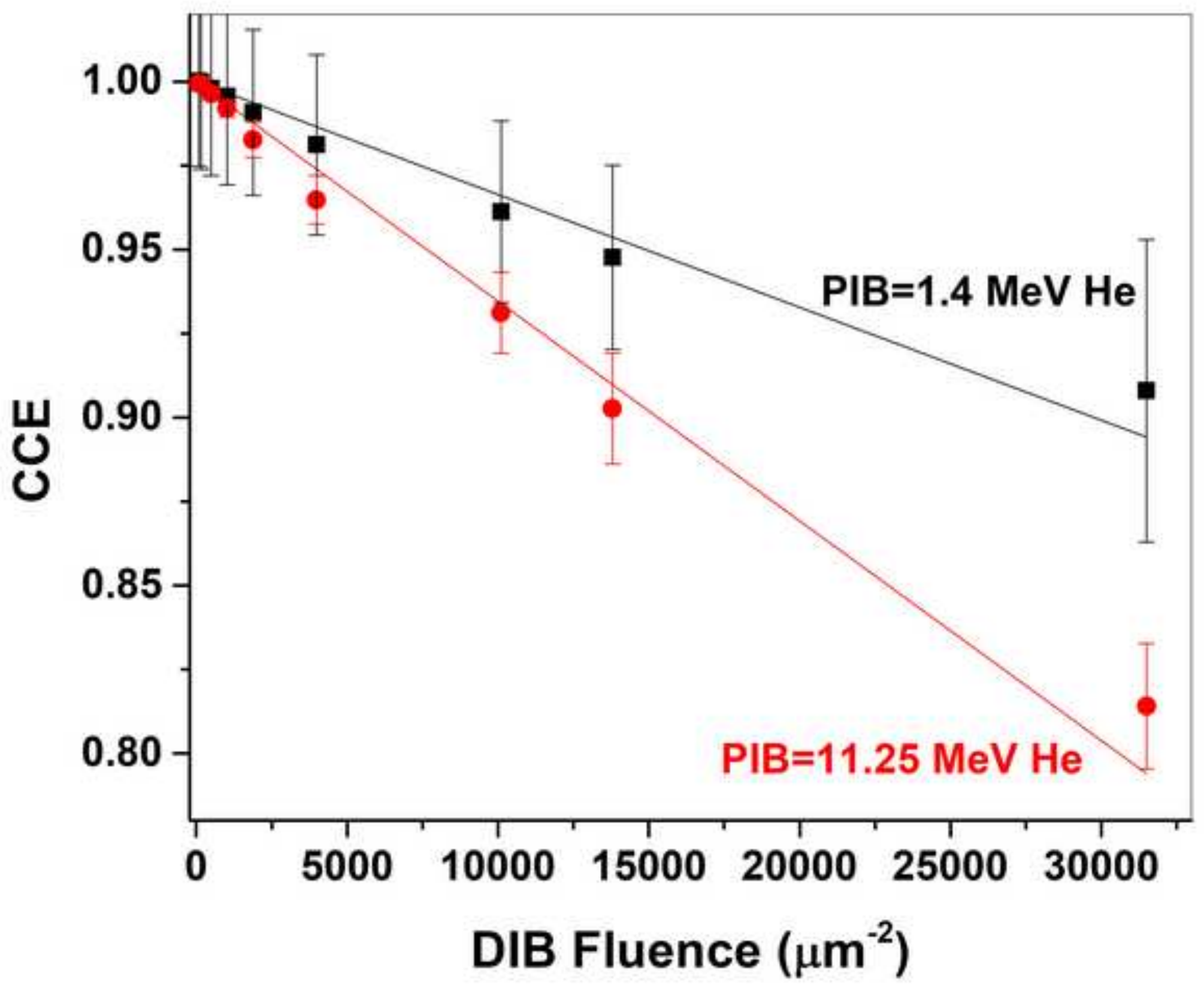




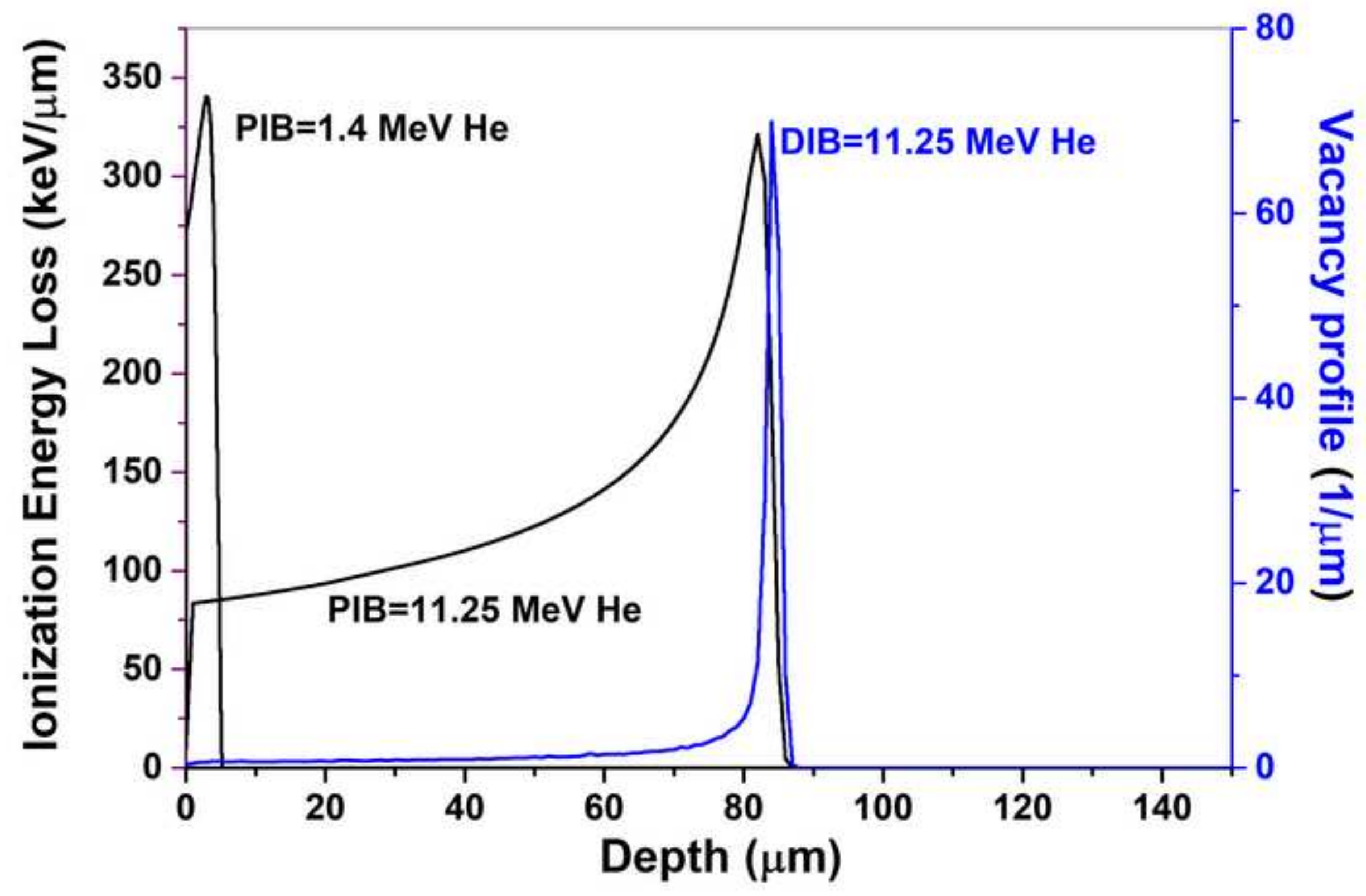


Click here to download high resolution image

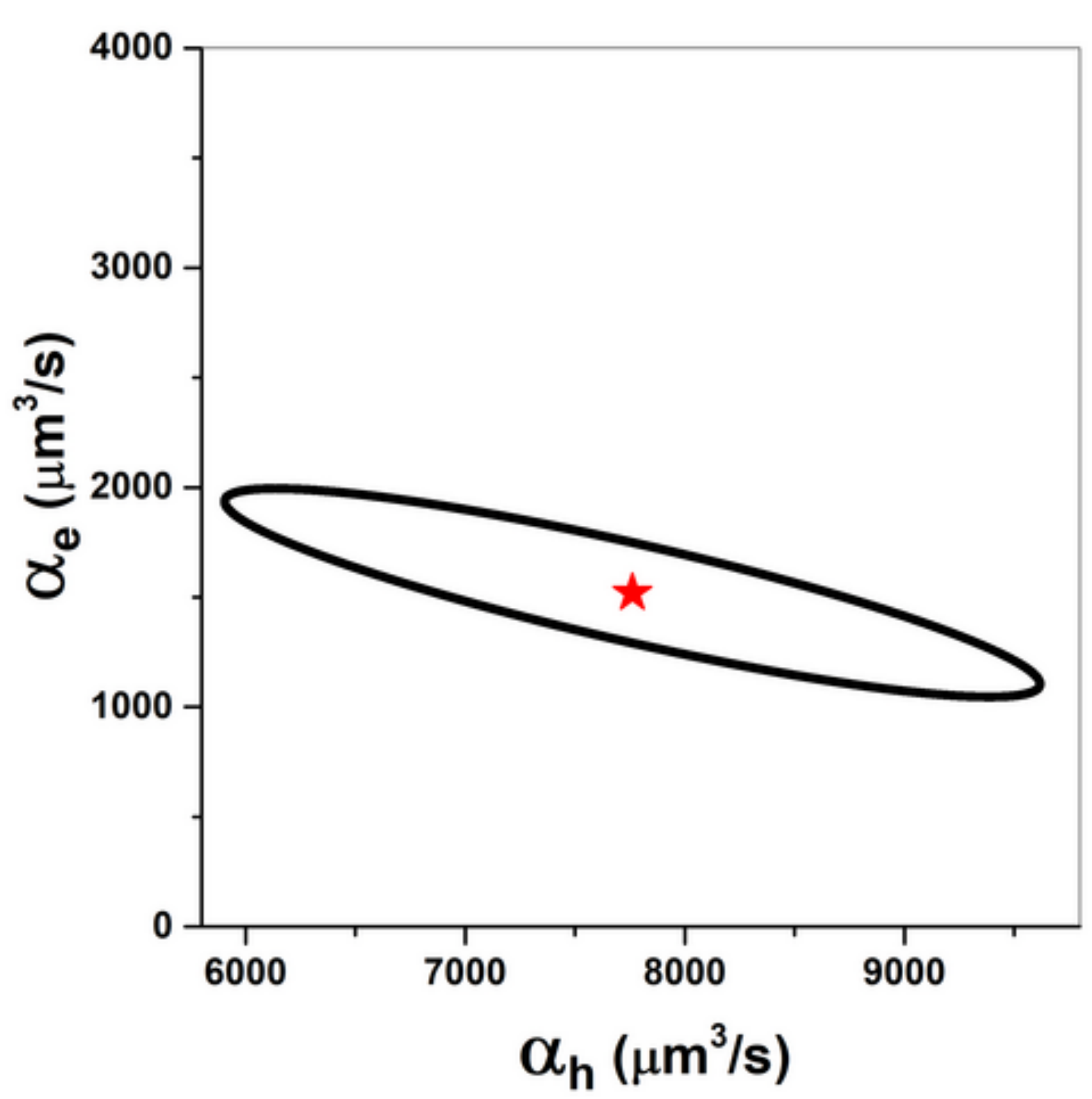

Click here to download high resolution image 